\title{
Experiencias significativas de Educación en Emergencia ${ }^{1}$
}

\section{Meaningful experiences of Education in Emergency}

\author{
Gustavo Antonio Zuluaga Trujillo ${ }^{2}$ \\ gustavosiglo21@gmail.com
}

\section{Resumen}

Desde hace más de sesenta años Colombia ha vivido violencias, incluyendo un conflicto armado interno. En el marco de este conflicto, la vida de muchos niños, niñas y adolescentes puede cambiar pasando de un estado de normalidad a otro de ruina, desorden, desolación, orfandad, angustia y en muchos casos padecer el desplazamiento y/o reclutamiento forzado. En este contexto de emergencia se ha evidenciado la incapacidad estatal para brindar seguridad y al mismo tiempo restablecer derechos a los sectores más vulnerables de la población, al igual que el irrespeto de lugares específicos que según el Derecho Internacional Humanitario no deben hacer parte de los conflictos porque son lugares para salvaguardar el derecho a la vida. La escuela y sus integrantes no están ajenos a estas situaciones, sin embargo, puede constituirse en escenario posible para rehabilitar el tejido social, devolver la esperanza, enseñar sobrevivencia y restituir derechos. En situaciones de emergencia mediados por conflicto armado, corresponde al Estado garantizar el restablecimiento de derechos entre ellos el de la educación de las niñas, niños y adolescentes. La intención del artículo es presentar los avances de una investigación respecto de las acciones pedagógicas y didácticas de maestras y maestros que han convertido su quehacer en experiencias significativas de educación en emergencia, con las cuales se propicia la reconstrucción social, la recuperación y el ejercicio de los Derechos Humanos porque la educación contribuye a transformar vidas.

Palabras clave: Escuela; Conflicto armado interno; Educación en emergencia; Derechos humanos; Experiencias significativas de educación en emergencia.

\begin{abstract}
For more than sixty years, Colombia has experienced violence, including an internal armed conflict. In the context of this conflict, the lives of many children and adolescents can change from a state of normalcy to one of ruin, disorder, desolation, orphanage, anguish and, in many cases, suffer displacement and / or forced recruitment. In this emergency context, the state's inability to provide security and at the same time restore rights to the most vulnerable sectors of the population has been demonstrated, as well as the disrespect of specific places that, according to International $\mathrm{Hu}$ manitarian Law, should not be part of conflicts because they are places to safeguard the right to life. The school and its members are not oblivious to these situations, however, it can be a possible scenario to rehabilitate the social fabric, restore hope, teach how to survive and restore rights. In emergency situations mediated by armed conflict the State must guarantee the restoration of rights, including the education of girls, boys and adolescents. The intention of the article is to present the progress of an investigation regarding the pedagogical and didactic actions of teachers who have turned their work into meaningful experiences of emergency education, with which social reconstruction, recovery and the exercise of Human Rights are promoted because education helps to transform lives.
\end{abstract}

Keywords: School; Internal Armed Conflict; Emergency Education; Human Rights; Meaningful Experiences of Emergency Education.

\footnotetext{
${ }^{1}$ Artículo de investigación.

${ }^{2} \mathrm{Mg}$. en Educación. Docente en la Institución Educativa José Cayetano Vásquez de Ciénaga. ORCID https://orcid.org/0000-0003-3171-0390

Asesora: Celina de Jesús Trimiño Velásquez.

${ }^{*}$ Cómo citar este artículo: Zuluaga, G. (2018). Experiencias significativas de educación en emergencia. Hojas y Hablas, (15), 49-65. DOI: 10.29151/hojasyhablasn.15a3
} 


\section{Introducción}

El presente texto es parte de un ejercicio investigativo que pretende mostrar la actividad educativa durante uno de los periodos más difíciles que ha producido el conflicto armado interno en Colombia. Uno de los procesos que más trastornos padece durante un conflicto armado es la educación; es por eso que en lo sucesivo se mostrarán acciones gubernamentales y no gubernamentales que aminoran el problema; a esas actividades se les denomina Educación en emergencia que para este caso son las emergencias educativas causadas por el conflicto armado interno.

El Estado Colombiano en el periodo 2002 - 2008; a través de la Organización de las Naciones Unidas, en adelante ONU, siguió una serie de líneas y trabajos coordinados por la Oficina de Cooperación Internacional, Colombia atravesaba por situaciones de emergencia desencadenadas por el conflicto armado y por ello requería colaboración internacional con el ánimo de superar la crisis en la que se encontraba.

La ONU ha organizado un sistema de trabajo con países que presentan características similares y que por voluntad propia decidieron cooperar para disminuir la crisis. En otro nivel jerárquico de la organización se encuentra la Mesa Nacional de Educación en Emergencia, organización de mayor instancia que para el efecto tiene el país, rodeada por otras instituciones de cooperación nacional e internacional como Save the children, oficina del Alto Comisionado de las Naciones Unidas para los Refugiados ACNUR, en inglés UNHCR, United Nations High Commissioner for Refugees, oficina de gestión del riesgo, el Ministerio de Educación Nacional y las Secretarías de Educación; acompañan también el proceso entidades privadas.
La mesa nacional de Educación en Emergencia tiene 18 miradas encargadas de dialogar, planear y diseñar estrategias técnicas, planes pilotos; entre las estrategias que debe dinamizar la mesa está contemplado el acompañamiento y fortalecimiento de las secretarías de educación con el ánimo de crear equipos de coordinación locales. El propósito de la educación en emergencia es desarrollar acciones que permitan disminuir las brechas en educación derivadas de algún tipo de crisis, garantizando espacios seguros, el derecho a la educación y proteger la dignidad de los menores involucrados por causa de la emergencia; para el caso de este estudio se trata de emergencias causadas por el conflicto armado interno que para la época señalada vivía Colombia.

Según la Organización Internacional para las Migraciones (OIM) la Educación en emergencia es un "proceso planeado que asegura la continuidad o restablecimiento de la educación en momentos de emergencia”. (Ministerio de Educación Nacional, 2012, pág. 3) Practicar el concepto implica conocer los riesgos existentes en el entorno escolar, estudiarlos, gestionarlos y fortalecer las instituciones educativas para que resistan adecuadamente los efectos de lo que puede ocasionar situaciones críticas como una emergencia o un desastre que no permita desarrollar las actividades académicas. Para el Ministerio de Educación Nacional (MEN) "La Educación en Emergencias (EeE) comprende desarrollos que permiten disminuir las brechas en educación derivadas de algún tipo de crisis, así como aquellos concernientes al acceso, permanencia, aceptabilidad y adaptabilidad en situaciones críticas" (Ministerio de Educación Nacional, 2011, pág. 7).

Dada la consideración anterior, es necesario diferenciar desastre de emergencia: emergencia es una situación que puede ser controlada por 
los entes territoriales, en este caso el MEN, y desastre es una situación que no puede ser controlada y requiere la intervención de un tercero. En el momento que atañe al estudio, que el país está en emergencia porque el conflicto armado está afectando a los ciudadanos y estos están siendo intervenidos por organismos internacionales con el ánimo de disminuir la vulneración del derecho a la educación.

Frente a la EeE hay dos enfoques, los cuales corresponden a definiciones como las expuestas por el MEN y la OIM: Enfoque de gestión del riesgo entendido como resiliencia o capacidad para auto gestionarse cuando se está en riesgo; y el enfoque de los derechos de las niñas, niños y jóvenes en edad escolar.

En el ámbito de la protección o restablecimiento de derechos es necesario plantearse preguntas que generen expectativas y respuestas claras que favorezcan a los más vulnerables en situaciones de emergencia:

1.¿Qué sucede con las niñas, los niños y los adolescentes durante una emergencia?

2.¿Por qué es importante el derecho a la educación durante una emergencia?

3.¿Quiénes deben garantizar el derecho a la educación en situaciones de emergencia?

De entre las muchas respuestas pueden destacarse las que se van a tratar en el presente artículo: los niños, las niñas y los adolescentes pasan desapercibidos, los niños no son priorizados para recibir bienes y servicios, son los más vulnerables, en muchos casos la escuela se convierte en un lugar seguro, el pronto retorno a clase significa sentimiento de normalidad para las vidas de las niñas, niños, jóvenes y sus familias la educación es un derecho aun en situaciones de emergencia y es clave para una vida con dignidad.
Se enuncian a continuación diferentes apreciaciones sobre el concepto de conflicto armado iniciando por lo acotado por el Derecho Internacional Humanitario (DIH) el cual hace una distinción entre dos tipos de conflictos armados, a saber:

-Conflictos armados internacionales, en que se enfrentan dos o más Estados

-Conflictos armados no internacionales, entre fuerzas gubernamentales y grupos armados no gubernamentales, o entre esos grupos únicamente. El derecho de los tratados de DIH también hace una distinción entre conflictos armados no internacionales.

-A los Convenios de Ginebra de 1949 y conflictos armados no internacionales, según la definición contenida en el artículo 1 del Protocolo adicional II. (Comité Internacional de la Cruz Roja (CICR), 2005)

Seguidamente y parafraseando a Uprimny en su texto Orden democrático y manejo de conflictos, analiza la Estructura y Dinámica del Conflicto en Colombia, argumenta sobre el tema diciendo que ha sido lo suficientemente dinámico de tal manera que, en 50 años de acontecimientos, ha pasado por varias fases y transformaciones diversas en las que puede destacarse la guerra de guerrillas, situación enmarcada en ideologías políticas de izquierda revolucionaria. Posteriormente, los colombianos apreciaron las políticas de diálogo y la voluntaria entrega de armas, con el patrocinio del Estado con normas de indulto que favorecían a los insurgentes que manifestaban su deseo de desmovilizarse para reinsertarse a la vida civil, social, política y productiva del país. (Uprimny, 2004, pp. 12, 13, 18)

Otros espacios destacados han tenido lugar en los diálogos de paz planteados por diferentes 
gobiernos y en distintas épocas, en los que un sin número de colombianos y miembros de la comunidad internacional tuvieron la oportunidad de pronunciarse, animando una salida pacífica al conflicto; en estas instancias han participado organizaciones académicas, permitiéndose sistematizar información relevante con respeto a las guerras internacionales e internas, con la finalidad de entender la naturaleza de los conflictos y hacer recomendaciones a otros países para que logren la paz.

En la mayoría de los casos, el fin de la guerra no ha llevado a finalizar las tensiones y el conflicto, al contrario, éstos se han incrementado a nivel interno y externo de las naciones. Muchos estados se encuentran en una frágil situación y riesgo de posibles conflictos futuros, especialmente cuando se atraviesa por problemas a nivel socioeconómico. Los niños en particular son víctimas de tales conflictos, ya que los espacios dedicados a desarrollar el proceso educativo son intervenidos para emplearlos como objetivos militares o barracas, y los niños y adolescentes son reclutados como combatientes, las niñas sufren violaciones, los profesores algunas veces son asesinados y los sistemas educativos son interrumpidos parcial o completamente.

Para ejemplificar el echo descrito en el párrafo anterior puede mencionarse que, en el 2001, el número total de refugiados se estimó en 15.8 millones (12 millones bajo el mandato de la ACNUR y 3.8 millones de Palestinos adicionales bajo el mandato de la Agencia de Naciones Unidas para la Ayuda a los Refugiados Palestinos en Oriente Próximo. Se estima que de aproximadamente 25 millones de personas a nivel mundial que han sido desplazadas internamente, la mayoría ha huido de conflictos, y solo una pequeña parte son desplazadas por desastres naturales, además de los millones de personas que han retornado a sus hogares en años recientes, quienes enfrentan las dificultades del proceso de reconstrucción de sus vidas; y hay millones más que viven en países o distritos inestables, donde la inseguridad ha producido la paralización de su sistema educativo. Además, cerca del 1\% de la población mundial, aproximadamente 50 a 60 millones de personas, viven bajo condiciones de emergencia o se encuentran bajo un proceso de reconstrucción sin que los niños, las niñas y los adolescentes tengan acceso a la educación. (CEPAL, 2001)

Según datos publicados por la ACNUR, Colombia tiene 4.7 millones de habitantes en el exterior. De esos nacionales que se fueron, 400.000 tienen el estatus de refugiado o lo están solicitando. Viven en 45 países del mundo. (El Tiempo, 2018)

En cuanto a los componentes de la EeE, El MEN dice: "La Educación en Emergencias (EeE) comprende las fases de prevención y gestión del riesgo, atención de crisis y emergencias, y post emergencia" (Ministerio de Educación Nacional, 2012, p.9). Los componentes de la EeE están enmarcados en el derecho a la educación. El Estado debe garantizar disponibilidad del servicio (la educación como elemento protector debe mantenerse o restablecerse lo antes posible), aceptabilidad (priorizar los más vulnerables, asegurar la atención diferencial y con equidad de género), accesibilidad (asegurar el acceso sin discriminación, tomando todas las medidas para cerrar las barreras de acceso y permanencia) y adaptabilidad (dar continuidad a los servicios sociales de atención psicosocial.

En cada una de las fases descritas hay unos actores que deben encargarse de brindar apoyo sicosocial, pedagógico, salud física y mental, 
protección y cuidado; a su vez en el ambiente de aprendizaje deben observarse aspectos como infraestructura, saneamiento básico, mobiliario y apoyo pedagógico, de la misma manera la institución educativa debe contar con el recurso humano que garantice el funcionamiento: directivos, docentes, administrativos y profesionales de apoyo.

En el periodo de estudio, Colombia vivió en medio de una crisis humanitaria como consecuencia de años de conflicto armado, especialmente con la acción de grupos insurgentes como las FARC (Fuerzas Armadas Revolucionarias de Colombia), ELN (Ejército de Liberación Nacional), y los grupos paramilitares; estos movimientos afectaron a la población de forma directa e indirecta convirtiendo algunos territorios en zonas de combate en los cuales también ha participado la fuerza pública, integrada por las Fuerzas militares y la Policía Nacional. Este fenómeno sigue conduciendo al país a un desplazamiento desde las zonas rurales a las urbanas que se llega a comparar con las peores crisis humanitarias a nivel mundial. Ya desde décadas atrás el fenómeno del conflicto venía traduciéndose en acciones contrarias al respeto por los derechos humanos, así lo dio a conocer Manuel Restrepo Yusti en uno de sus escritos:

las autoridades gubernamentales han venido sosteniendo quelas dificultades para mejorar la situación de derechos humanos derivan en gran parte de las acciones terroristas de la guerrilla, como fuerza históricamente responsable de la violencia y en años posteriores del surgimiento del narcotráfico. (Restrepo, 2008, p.105).

El conflicto colombiano, tiene antecedentes históricos en la violencia; inicia con el conflicto bipartidista de la década de 1.950, en la décadas de los años 60 y 70 surgen las guerrillas insurgentes y subgrupos al margen de la ley, el problema se incrementó en 1988, cuando estaba en la presidencia Virgilio Barco Vargas gobierno con el cual se rompieron los diálogos de paz con las guerrillas, posteriormente en el gobierno de César Gaviria Trujillo nuevamente se consolidan diálogos de paz, también hubo diálogos propiciados por el gobierno de Andrés Pastrana. En el inicio de la presidencia de Álvaro Uribe Vélez las cifras del desplazamiento y crisis humanitaria no disminuyeron como se había previsto, cerca de tres millones de personas fueron desplazados internos, los homicidios políticos por año aumentaron de 3000 a 6000; situaciones que sumieron al país en la pobreza y el desarraigo.

De acuerdo con el Banco Mundial (2007), con un intervalo poco amplio entre la pobreza a comienzos del conflicto en la década de los años 50, 52\%; con relación al periodo de estudio, casi 49.2 \% de la población se mantenía en la pobreza. El conflicto armado en el país ha dejado variedad de consecuencias; como la interrupción de las formas de subsistencia, desarraigos, secuestros, asesinatos y otras formas de violación a los Derechos Humanos, mientras el Estado ha generado políticas nacionales y ha adoptado o imitado acciones internacionales que propenden por su protección.

El sector educativo, liderado por el MEN y otras organizaciones de ayuda humanitaria de carácter gubernamental y no gubernamental; han intentado hacer frente a las problemáticas mejorando la infraestructura, ampliando la cobertura y propiciando calidad del servicio educativo para las poblaciones en condición de vulnerabilidad, incluyendo a los desplazados. Sin embargo, las memorias del seminario y reuniones de sistematización del Grupo Gestor de la educación en situaciones de emergencia en Colombia mencionan: 
En muchas de las organizaciones humanitarias, quizás por el carácter intangible de los efectos de la educación, no se ha reflexionado frente al hecho de que una educación pertinente y de calidad se constituye, no sólo en una adecuada herramienta de protección y prevención, sino en la mejor opción para enfrentar la crisis de manera organizada e inteligente (Ministerio de Educación Nacional, 2008, p. 2)

Es así, como la educación, más allá de ser un mero servicio público, se convierte en un instrumento de restablecimiento, reparación y reconstrucción de los recursos humanos y físicos que han sufrido el impacto directo del conflicto armado. A causa de ello, la educación tiene un potencial extraordinario como herramienta para la construcción de la paz y al tiempo para instigar violencia. Por esta razón, el Relator Especial de la Organización de las Naciones Unidas (ONU) sobre el derecho a la educación lo ha expuesto en Fundación Dos Mundos de la siguiente manera:

La educación puede jugar un papel fundamental en la promoción de la cooperación y el entendimiento humano. Pero al mismo tiempo, un tipo de educación que no construye la paz, sino que acrecienta las desigualdades sociales y de género, lejos de ser benigna, puede más bien confabular con el conflicto. (Muñoz, 2008, p. 8)

En efecto, con los riesgos que se enfrentan ante un conflicto, el ámbito escolar es un lugar privilegiado para la transformación de la cultura y junto con la familia, constituye los escenarios para la construcción de reglas propicias al ejercicio democrático del poder y del encuentro social, el fortalecimiento de las habilidades ciudadanas y la promoción de un entorno protector y promotor de bienestar emocional.
La educación es, pues, un fin en sí mismo, como una herramienta para el logro de otros fines, puede desempeñar un papel que resguarde la vida y la integridad física de niños y niñas, hacer posible su protección psicosocial y garantizarles un proceso cognitivo que facilite su futuro. A partir de su potencial de favorecer mecanismos de protección, la educación resguarda y sustenta las vidas de los educandos. De esta manera la educación como proceso se vale de la escuela, cuando garantiza un espacio protector de la violencia y forma a los estudiantes para asumir decisiones informadas y seguras frente a eventuales riesgos, es un espacio propicio para protegerlos de la explotación y del abuso, particularmente de la violencia por razones de género, la explotación sexual y su vinculación a grupos armados de origen legal u organizados al margen de la ley; así mismo, previene su participación en actividades ilícitas o conformación de pandillas. En contextos de conflicto armado se les prepara para enfrentar situaciones derivadas del mismo, como ataques a la infraestructura escolar, accidentes por minas anti personas, violencia sexual, VIH-SIDA y otras enfermedades de transmisión sexual.

A través de la garantía del derecho a la educación puede proveerse bienestar psicosocial a niños y niñas que viven en medio de situaciones de conflicto armado. En términos generales, la escolaridad les brinda un sentimiento de pertenencia, de normalidad, estructura y estabilidad, aun en el marco de situaciones extremas como la violencia armada. Igualmente es relevante para su protección psicosocial; así lo afirma la Fundación Dos Mundos "la posibilidad que la educación brinda de socializar, establecer o fortalecer redes sociales con pares, donde puedan dialogar, reflexionar y brindarles apoyo mutuo, así como desarrollar sentimientos de valía propia, confianza y esperanza en el futuro". (Fundación Dos Mundos, 2009, 
p. 23); entre otros aspectos el estudio señala que la existencia de una rutina diaria - al propiciar ritmos y responsabilidades - permite promover bienestar psicosocial y favorece el restablecimiento de un sentido de estructura y propósito en la vida, que puede verse afectado por los hechos de violencia asociados al conflicto.

Debe destacarse que el Estado tiene una responsabilidad directa no solamente con la planeación y entrega del servicio a la educación, sino también a que ésta responda de forma temprana y coherente a las necesidades de la población en general, y más aún, de aquellas que se encuentran en condiciones de vulnerabilidad, lo cual se logra teniendo pleno conocimiento de las propias comunidades y a su vez de las experiencias que en otros países se han desarrollado con éxito en torno a la educación en situaciones de emergencia originadas específicamente por el conflicto armado.

En el campo legislativo internacional e independientemente del contexto político, social o económico en el cual se desarrolle el individuo, éste debe estar en plena facultad de gozar el disfrute del derecho fundamental a la educación. En primer lugar, la Declaración Universal de Derechos Humanos, en su Artículo 26을 numerales 1 y 2 cita:

a.Toda persona tiene derecho a la educación. La educación debe ser gratuita, al menos en lo concerniente a la instrucción elemental y fundamental. La instrucción elemental será obligatoria. La instrucción técnica y profesional habrá de ser generalizada; el acceso a los estudios superiores será igual para todos, en función de los méritos respectivos.

b. La educación tendrá por objeto el pleno desarrollo de la personalidad humana y el fortalecimiento del respeto a los derechos humanos y a las libertades fundamentales; favorecerá la comprensión, la tolerancia y la amistad entre todas las naciones y todos los grupos étnicos o religiosos; y promoverá el desarrollo de las actividades de las Naciones Unidas para el mantenimiento de la paz. (Naciones Unidas, 2015)

Según los preceptos anteriores, el abordaje de políticas y programas orientados hacia la cobertura, gratuidad y obligatoriedad de la provisión educativa para un Estado, debe considerarse no como un privilegio del cual son o no merecedores los ciudadanos; sino por el contrario, como una obligación que a nivel mundial debe cumplirse y acatarse.

En el contexto colombiano, el derecho a la educación se expone en la Constitución Política de Colombia de 1991, específicamente dentro de los derechos sociales, económicos y culturales en sus Artículos 44, 45 y más específicamente en el Artículo 67, el cual resalta la importancia de la formación en el respeto a los derechos humanos, la paz, la democracia y de la obligación del Estado de velar por la cobertura del servicio, la calidad y las condiciones que aseguren el acceso y permanencia en el sistema educativo. (Corte Constitucional, 2016) Ahora bien, en el año 2006, se expide la Ley 1098 noviembre 8 de 2006, Código de la Infancia y la Adolescencia, con el objeto de orientar al establecimiento de normas para la protección integral de los niños, las niñas y los adolescentes cuya responsabilidad se adjudica explícitamente a la familia, la sociedad y el Estado. En sus artículos 17, 18, y 19 se brindan elementos legales frente al derecho a la vida, a la calidad de vida, a un ambiente sano, a la integridad personal y a la rehabilitación y la resocialización, relacionados cada uno de ellos con la educación y otros derechos fundamentales. (Procuraduría General de la Nación, 2010) 
En cuanto a esta Ley, es importante destacar que es una de las primeras muestras, en términos legales expuestos en la situación de conflicto armado y las consecuencias directas que éste ha tenido sobre la población civil, y aún más específicamente sobre los menores de edad. Sin embargo, a su vez se representa la grave crisis que enfrenta el país en términos de protección a la infancia, pues describe los hechos de los cuales son víctimas en su mayoría los menores de edad, que han estado lesionados total o parcialmente no sólo en los derechos consagrados en el código sino en aquel considerado como fundamental, el derecho a la vida.

Continuando con el aporte legal, en este apartado, se realiza una descripción de las leyes, decretos y políticas educativas que a nivel nacional se han desarrollado con el fin de mejorar la prestación del servicio educativo en diferentes ámbitos, tales como pertinencia, calidad y cobertura, entre otros.

En 1994, la Ley General de Educación, cuyo objeto refiere a la educación como un proceso de formación permanente, personal, cultural y social que se fundamenta en una concepción integral de la persona humana, de su dignidad, de sus derechos y de sus deberes, en su Artículo 5, estipula entre otros fines:

El pleno desarrollo de la personalidad sin más limitaciones que las que le imponen los derechos de los demás y el orden jurídico, dentro de un proceso de formación integral, física, psíquica, intelectual, moral, espiritual, social, afectiva, ética, cívica y demás valores humanos. La formación en el respeto a la vida y a los demás derechos humanos, a la paz, a los principios democráticos, de convivencia, pluralismo, justicia, solidaridad y equidad, así como en el ejercicio de la tolerancia y de la libertad. (Congreso de la República de Colombia, 1994)
El marco antes expuesto, genera, entre otras, ésta pregunta generadora es: ¿Cuáles son las experiencias significativas (los hechos y acciones realizadas por diferentes organismos nacionales e internacionales) que sustentan la investigación en Educación en Emergencia dada en el marco del escenario del conflicto armado en Colombia, en el período 2002 - 2008? Responder esta pregunta, orientará el desarrollo de esta investigación con una mirada pedagógica y social. El cuestionamiento es el punto de partida teórico para todos aquellos expertos comprometidos con la educación de calidad y especialmente con la reflexión y desarrollo de estrategias para la Educación en Emergencia como ámbito generador de experiencias educativas para gestionar la paz desde la escuela.

No son muchos los documentos investigativos sobre Experiencias Significativas de Educación en Emergencias que abarquen con rigor exploratorio los enfoques y paradigmas propios de este tipo de investigación; fue necesario elaborar un estado del arte que reuniera, clasificara e interpretara información que a nivel colombiano, y experiencias internacionales se produjeron durante el periodo de estudio; esto marcó un inicio claro, sólido y determinante para todos los estudios de indagación en EeE actuales y posteriores a esta investigación. De esta manera, la investigación como resultado de esta actividad investigativa, puede comprometerse con la elaboración de documentos e insumos útiles para organizaciones gubernamentales y no gubernamentales, interesadas en la divulgación, promoción, diseño y evaluación de planes y prácticas de Educación en Emergencia por conflicto armado.

Los objetivos están encaminados hacia la revisión de documentos existentes e información pertinente que permita el desarrollo metodológico de la investigación y la elaboración del estado 
del arte, tomado como punto de partida una mirada crítica a procesos de investigación rigurosos basados en historiales y experiencias significativas de la población y objeto de estudio; así pues se trata de presentar las experiencias significativas (los hechos y acciones realizadas por diferentes organismos de educación) en el panorama colombiano de la educación en emergencia en el marco del escenario del conflicto armado, especificando el impacto del conflicto armado en el disfrute efectivo del Derecho a la Educación y las estrategias para el restablecimiento de este derecho, de acuerdo al estudio investigativo; además de identificar las estrategias usadas para la reconstrucción del tejido social encontradas a través del estudio realizado en educación en emergencia.

Muchos aspectos que caracterizan el conflicto y la violencia durante el periodo de estudio siguen siendo los mismos desde que inició a mediados del Siglo XX, Chaparro Amaya en su libro Génesis y Transformaciones del Estado Nación en Colombia; analiza desde la promulgación de la Constitución del 1991 el afianzamiento de una nueva sociedad que ha llevado a la práctica algunos derechos para instaurar una mejor forma de vida en comunidad, aclarando que la ciudadanía ha tenido la voluntad de asumir estos procesos; pero ciertas fuerzas del Estado o al margen de la ley desacatan la norma, violentando los derechos fundamentales, menoscabando el patrimonio $y$ los bienes que la justicia debería proteger. (Chaparro, 2009)

Desde el punto de vista de la teoría; la educación en emergencia no ha ocupado tradicionalmente un lugar importante en el pensamiento humanitario; otras amenazas a la vida como la falta de comida, el refugio o el cuidado de la salud, pueden ser a primera vista más importantes. Los programas educativos que se encuentran en ambientes de conflicto y crisis, han sido vistos como un lujo, y como un tema que desarrolla mejor la comunidad. Esto ha llevado a reconocer que los Estados están obligados a aceptar ayuda en el establecimiento de las necesidades educativas de los niños y adolescentes afectados por la emergencia en los casos en que no se encuentren en posición para encontrar dichas necesidades.

El término "educación en emergencias se refiere a la educación formal y no formal en situaciones donde los niños pierden el acceso al sistema educativo nacional y de su comunidad debido a emergencias complejas o desastres naturales" (Triplehorn, 2001, p.3) Como consecuencia del nivel de frecuencia, a la larga duración y a que los conflictos modernos son crónicos por naturaleza, la palabra "emergencia" se refiere no sólo a programas iniciales de educación informal y no formal, también denota el establecimiento de programas de educación formal durante el conflicto; entendiendo la educación informal como un proceso de aprendizaje dado a través de las experiencias cotidianas y de la transferencia de conocimientos, habilidades y actitudes a partir de la tradición cultural, familiar, comunitaria y de los medios de comunicación; la educación no formal como una aproximación flexible a la educación empleando vías alternativas de desarrollo fuera del sistema formal cuyos contenidos, pueden ser idénticos a los que están disponibles en la escuela o pueden ser diferentes, como en el caso de programas de alfabetización e iniciativas de educación popular que no llevan a una certificación.

La educación se convierte en una herramienta de reconstrucción del tejido social; es un componente fundamental de la ayuda humanitaria en contextos de emergencia. La Agencia de los Estados Unidos para el desarrollo internacional (USAID), Save the Children, el Fondo de las 
Naciones Unidas para la Infancia (UNICEF) y la Organización de las Naciones Unidas para la Educación la Ciencia y la Cultura (UNESCO) promovieron modelos flexibles para formar promotores comunitarios capaces de atender las necesidades educativas de niños en educación primaria y preprimaria en Nicaragua, Guatemala, El Salvador, Uganda y Afganistán. (Malley \& Triplehorn, 2005, p.14)

Son estas las instituciones que más documentos han producido documentos y herramientas propias del tema de estudio además de diversas ONGs, como: Fundación Dos Mundos, Comité Internacional de la Cruz Roja (CICR), Red interinstitucional para la Educación en Situaciones de emergencia (INEE) (Minimum Standards for Education in Emergencies), y la Plataforma Colombiana de Derechos Humanos, han desarrollado estrategias de ayuda en: educación para niños excombatientes, alfabetización, habilidades para la vida, reintegración de niños soldados, educación técnica y vocacional, agricultura y formas de sustento, programas de educación acelerada y servicios comunitarios de aprendizaje, entre otras; y a la vez, diferentes equipos de investigación, como el Alto Comisionado de las Naciones Unidas para los Refugiados (ACNUR), la organización de las Naciones Unidas (ONU), SAVE THE CHILDREN mencionan acerca de los proyectos, críticas de cómo las ONGs que encabezan estos contextos no utilizan los medios y mecanismos adecuados para tratar de solucionar las necesidades de la población desplazada y se presentan casos donde son cerrados injustamente.

Actualmente, hay gran cantidad de tendencias importantes en el camino de la ayuda para el desarrollo; desde el extranjero las asociaciones están acercándose a los donantes, agencias internacionales y sociedad civil (éstas no son mutuamente excluyentes) desarrollan el enfoque de impulsar la inversión fundamentada en los problemas que apuntan a necesidades específicas. Consecuentemente, las asociaciones globales están formadas para atacar un tema de desarrollo específico y demostrar resultados. (Sibbons, 2004)

Es ampliamente conocido que durante y después de un conflicto armado la población civil es la más afectada, y dentro de ésta, los niños, niñas y adolescentes se encuentran más vulnerados y violados en sus derechos fundamentales. Inicialmente se pueden describir dos formas en que esta población se vincula con la guerra; la primera, en donde los menores son víctimas directas del conflicto, sufriendo las consecuencias de la destrucción, el abuso, la pérdida de la protección más elemental, los perjuicios para su salud física y mental y el derecho a la educación, así como el desplazamiento territorial forzoso producto de la violencia; la segunda, la que configura la violación más flagrante de sus derechos, trata del reclutamiento de niños y adolescentes como parte de los cuadros combatientes o como parte de los grupos de apoyo a las tropas durante las confrontaciones. (ONU, 2005)

Asimismo, es necesario prever, el alto costo económico y social de los conflictos en cualquier nación que los sufra, estos eventos se extienden por años o décadas; tal como ocurre en Colombia y en otros países del mundo; por consiguiente, los mecanismos de atención a la población desplazada deben ser planificados como proyectos a largo plazo que ameritan ir más allá de la mera asistencia y concentrarse en la prevención, atención humanitaria y restablecimiento de los derechos fundamentales de las poblaciones afectadas. (Ministerio de Educación Nacional, 2004) 
En medio del conflicto los niños constituyen parte de la población más vulnerable, es incalculable el daño físico y psicológico que pueden sufrir estos seres humanos debido a su dependencia y debilidad frente a los adultos. Específicamente en Colombia, muchos niños son separados de sus padres y obligados a formar parte de grupos armados, otros están expuestos a ser blanco de la violencia o minas terrestres, algunos mueren en combate o quedan mutilados por los ataques de los grupos insurgentes a la población civil, por esta razón las escuelas son destruidas y los profesores asesinados, torturados o son víctimas de amenazas motivo por el cual deben abandonar sus lugares de trabajo.

En Colombia, esta situación se ha prolongado a lo largo de 6 décadas y la intensificación de los combates ha incidido en la incorporación de niños a las filas de los grupos armados como: las Fuerzas Armadas Revolucionarias de Colombia (FARC), el Ejército de Liberación Nacional (ELN) y las Auto Defensas Armadas de Colombia (AUC) (Human Right Watch, 2002, p.19) ,en calidad de colaboradores e informantes, siendo esta situación la generadora de una emergencia en cuanto al derecho fundamental de la educación se refiere. Dado que el periodo del conflicto es extenso, la investigación recae temporalmente hablando en el periodo de gobierno del presidente Uribe Vélez para detallar hechos y acciones que favorecieron la EeE realizados en el país durante el mencionado periodo dado que fue en esta época que el conflicto armado colombiano tuvo su periodo más crítico en cuanto a violación de derechos humanos se refiere.

En Colombia las sistemáticas violaciones de los derechos humanos, las infracciones al Derecho Internacional Humanitario (DIH) y otras formas de violencia sociopolítica e intolerancia social, configuran, entre otros, algunos elementos de la crisis humanitaria por la que atraviesa el país. Es así que, las consecuencias del conflicto y de las diversas violencias aplicadas en contra de la población civil, en particular aquella sometida a procesos de expulsión, destierro, despojo, despoblamiento y eventual repoblamiento, son profundas y prolongadas, e incluso abarcan varias generaciones que han tenido que afrontar en distintos momentos de su vida el desplazamiento forzado.

Los principales donantes internacionales se adhieren al enfoque funcional-estructural que busca fortalecer la debilidad y fragilidad del estado colombiano a través de paquetes educativos de emergencia. Consecuentemente, ellos han sido muy lentos en criticar al gobierno colombiano por su terrible registro de violación de los Derechos Humanos y han sido renuentes a ejercer toda su influencia para modificar su comportamiento.

En cuanto al compromiso de la comunidad internacional con las comunidades, gobiernos locales y el Estado colombiano en su conjunto han posibilitado la cooperación internacional, en muchos casos soporta el trabajo de entidades públicas y privadas en programas de prevención, atención humanitaria, restablecimiento de derechos $\mathrm{e}$ integración.

En Colombia, la mayoría de los niños que se unen a la guerrilla o los paramilitares, proceden de entornos muy pobres, donde carecen de cualquier oportunidad de educación, progreso o estatus social; muchos de ellos abandonaron la escuela antes de completar la primaria. Todos los niños tienen historias diferentes sobre las razones que le llevaron a incorporarse a la guerrilla, pero en casi todos los casos, la decisión fue provocada por varios factores como la pobreza, las privaciones, el subempleo, la escolarización truncada, la falta 
de afecto y apoyo familiar, los malos tratos de los padres y la inseguridad.

La utilización de niños y adolescentes en conflictos armados pone a los menores en situaciones de alto peligro para su vida, integridad y educación; en las investigaciones trabajadas en la documentación se evidencia que los estudiantes por sus condiciones de vida optan por ingresar a las filas de los grupos armados que operan en la zona, las escuelas son utilizadas por los grupos armados como sitios de reunión o de resguardo de la confrontación armada, afectan el rol del maestro y generan temor en ellos. En general, las comunidades con predominio rural saben que los niños, niñas y jóvenes son vinculados a los grupos armados al margen de la ley, sin embargo, en las ciudades se evidenció que este tema no era visible para ellos y la realidad de la situación de los niños, niñas y jóvenes en zonas de conflicto era lejana Fundación Dos Mundos (2009).

La Corte Constitucional ha puntualizado que todos los actos de política pública deben estar dirigidos a la reparación moral, material y simbólica de los daños ocasionados a las personas en situación de desplazamiento, de conformidad con el ordenamiento constitucional, las leyes y decretos pertinentes, y los Principios Rectores de los Desplazamientos Internos. El marco normativo de protección de la población desplazada interna no se agota entonces en la legislación nacional colombiana, toda vez que, de acuerdo con la jurisprudencia de la Corte Constitucional, los principios rectores de los desplazamientos internos y la normatividad internacional relevante, como los recientemente aprobados principios sobre la restitución de las viviendas y el patrimonio de los refugiados y de las personas desplazadas, hacen parte del bloque de constitucionalidad y de la inclusión educativa. Ministerio de Educación Nacional (2008)
La Plataforma Colombiana y la Interamericana de Derechos Humanos, Democracia y Desarrollo deben continuar promoviendo en el sistema de Naciones Unidas el debate por el sostenimiento del tratado internacional que permite a las víctimas de violaciones de los derechos económicos, sociales y culturales (DESC); se trata del Protocolo Facultativo del Pacto Internacional de Derechos Económicos, Sociales y Culturales en adelante (PF - PIDESC) el cual permite presentar denuncias a nivel internacional cuando las personas no pueden acceder a la justicia en los tribunales de su país por violaciones a estos derechos (DESC). El PF - PIDESC, aporta herramientas para la presentación de casos ante la violación de los derechos económicos, sociales y culturales y en particular del derecho a la educación.

Se espera que Colombia avance en la construcción de políticas públicas de educación en emergencia, a través de acciones de abogacía y movilización, incluyendo la reactivación de la mesa nacional sobre educación y el impulso a mesas regionales similares; el desarrollo conjunto de experiencias piloto regionales, que permitirán la formulación conjunta de unos indicadores mínimos que faciliten los procesos de monitoreo, seguimiento y evaluación a la atención educativa de las emergencias y acciones de comunicación y divulgación. (Weiss Fagen, Fernández Juan, \& Stepputate, 2009).

La educación es prioridad durante una emergencia porque los niños y su futuro están en riesgo, los niños tienen un derecho fundamental; se trata de la educación y los programas educativos en emergencias deberían proteger o cumplir con este importante derecho, incluso durante el conflicto; además para apoyar el desarrollo social e intelectual de los niños, los programas educativos pueden brindar protección física de los peligros de un ambiente en crisis, mitigando el impacto psico- 
social de la crisis, y facilitando la identificación de los niños que necesitan asistencia especial.

En cuanto a la profundidad de la investigación, tomando como base la propuesta metodológica expuesta por González, se enmarca en los siguientes postulados:

Esta se enmarca en el nivel exploratorio descriptivo, siendo la finalidad del primer objetivo examinar o explorar un tema o problema de investigación poco estudiado o que no ha sido abordado nunca antes. Por lo tanto, es útil para acercarse a fenómenos relativamente desconocidos, poco estudiados o novedosos y a la vez nos permite identificar conceptos o variables promisorias, e incluso identificar relaciones potenciales entre ellas. (González, 2005, p. 112)

A su vez el nivel descriptivo, permite la selección de cuestiones, conceptos o variables y se mide cada una de ellas independientemente de las otras, con el fin precisamente de describirlas; se pretende especificar las propiedades importantes de personas, grupos, comunidades o cualquier otro fenómeno. Por consiguiente, los estudios descriptivos pueden ofrecer también la posibilidad de hacer predicciones incipientes, aunque sean rudimentarias (Hernández, R. \& Fernández, C, 2014)

En cuanto al alcance de esta investigación sobre Educación en Emergencia permite en principio delimitar y caracterizar el campo de estudio objeto de la investigación y su impacto real tanto en el avance de dicho estudio como en la comunidad educativa, detectar los problemas recurrentes o relevantes en el proceso de desarrollo investigativo, estudiar los tratamientos conceptuales, los enfoques teóricos y metodológicos de los problemas, contextualizar la Comunidad investigada, los grupos, las líneas y los proyectos de investigación en su proceso histórico y en el momento actual, hacer un balance de los vacíos y debilidades (metodología, problemas, marco conceptual) con mirada crítica y visión de futuro de un campo investigativo. 2008.

1.Delimitación temporal: periodo 2002-

2.Delimitación geográfica: Colombia.

3.Selección de los informes más representativos generados por los Organismos nacionales e internacionales que desarrollen acciones significativas en el campo de acción en emergencias.

4.Selección de textos con descriptores bibliográficos editados y financiados por entidades sociales o educativas.

Para el análisis de la información y el logro de los objetivos de la investigación, se hizo necesario llevar a cabo el siguiente trayecto metodológico: un primer momento para establecer la definición de la situación problema; seguidamente la ubicación, clasificación, lectura y reseña de documentos. Por reseña se entiende el ejercicio de revisión o examen que se hace a un libro o a una obra para dar noticia crítica de sus argumentos. En un tercer momento se seleccionaron categorías conceptuales vistas desde los argumentos teóricos proporcionados por los autores estudiados. Una cuarta parte en la cual se sistematice la información en forma de matrices conceptuales, de acuerdo a la teoría de análisis del discurso propuesta por Van Dijk con el fin de apoyar la comprensión e interpretación final de los textos. (Van Dijk, 1999) y un quinto y último momento en el cual se dé la producción del informe final primero para presentarlo a evaluación para el examen de suficiencia y después la tesis doctoral.

Como criterios relevantes para la selección de la muestra textual, se formularon los siguientes: marcos teóricos o resultados de investigación y experiencias sobre Educación en Emergencia, textos que registran experiencias relevantes a nivel internacional, publicaciones en el periodo comprendido entre los años 2002 y 2008 y testimonios de comunidades educativas que padecieron el conflicto armado en Colombia durante el mismo lapso de tiempo. 
En Colombia, la revista "Más allá del Embrujo" destaca acciones importantes del tercer año de gobierno de Álvaro Uribe Vélez, resalta que "la educación es pilar fundamental para desarrollar procesos que generen equidad y ayuden a construir ciudadanías más conscientes y activas" (CINEP, 2005, p. 94) disminuyendo las brechas de pobreza, el estado debe garantizar la educación como un derecho fundamental que favorezca a los habitantes más vulnerados socioeconómicamente.

Teniendo en cuenta las consideraciones producidas por el informe mencionado en el párrafo anterior; el estado colombiano y desde la mirada de la educación en emergencia por el conflicto armado ha de procurar avances significativos, debe preocuparse por dinamizar políticas públicas encaminadas al mejoramiento de la calidad de la educación mediante la cualificación de los docentes, construcción de edificios nuevos, dotación de laboratorios, programas de bilingüismo y tecnología, escolaridad desde los cuatro años, y programas de alimentación escolar como práctica para disminuir la deserción. A pesar de los esfuerzos la educación aún no llega a todos los sectores, especialmente a los afectados por el fenómeno del desplazamiento, debido a que son muchos y están dispersos por todo el territorio nacional. (Plataforma Colombiana de Derechos Humanos Democracia y Desarrollo, 2010)

Susan Nicolai en el texto Oportunidades para el cambio aprueba la alfabetización como una situación específica abarcadora que fomenta la reconstrucción posconflicto, es una oportunidad para mejorar las capacidades de las naciones, esta clase de educación permite la flexibilización curricular enfocando el desarrollo de las clases en hechos que conduzcan al desarrollo de una paz sostenible, la práctica de los derechos humanos; de esta manera, la alfabetización favorece también la participación de las mujeres y niñas y se convierte en un medio de reinserción en la vida civil. (Nicolai \& Hine, 2015)
El mismo documento referencia el paquete educativo como una oportunidad de inclusión educativa para las zonas apartadas de los pueblos en conflicto, un sin número de los desplazados buscaba las zonas rurales más apartadas para huir de los actos belicosos. Las ONG (Comité Internacional de la Cruz Roja CICR) y la INNE, Normas Mínimas para la Educación en Emergencia) preocupadas por esta población tan vulnerable propusieron los paquetes educativos, los cuales incluían un docente capacitado y equipado con un kit escolar que le permitiera desplazarse hasta los lugares más apartados. Esta estrategia dio buenos resultados, pero en los lugares en los que se agudizó el conflicto se dejó de promover por el riesgo que corrían los docentes y la reunión de grupos de más de diez personas que podrían ser objeto de reclutamiento. (INNE, 2004)

La investigadora Nicolai comenta también que la educación técnica y de desarrollo de habilidades para la vida son una oportunidad para sacar del conflicto a la población menor de 18 años y posibilitar la participación de las mujeres y las niñas en la vida escolar ofreciéndoles aprender un oficio que les permitiera, inclusive obtener algunos dividendos o iniciar algún negocio. Otro aspecto importante es la necesidad de cualificar los docentes para que estén preparados o tengan idea de cómo actuar en momentos de crisis. Es así que, muchos gobiernos y las organizaciones internacionales han emprendido programas para fortalecer la educación desde los educadores (2015, p. 28).

Se puede concluir diciendo que los enfoques de la Educación en Emergencia para la sociedad civil en situaciones de conflicto armado se dirigen a dar respuesta específicamente a las necesidades de supervivencia básicas, tales como el reconocimiento de minas anti persona, desarrollo de habilidades para el sustento y prevención de abuso sexual, transmisión de enfermedades y del resurgimiento de nuevas tensiones entre los actores del conflicto. 
De igual forma, las dinámicas de los conflictos armados traen consigo oportunidades para restablecer y encaminar de mejor manera el sistema educativo, pues como respuesta a la crisis se han replanteado políticas y currículos educativos que impulsan el desarrollo de programas de educación formal y no formal, orientados hacia el desarrollo de habilidades y destrezas, metodologías sensibles al género, y programas de aceleración y alfabetización de niños, jóvenes y adultos, los cuales contribuyen a la rehabilitación de la población afectada.

Las asociaciones, entre las ONG, gobiernos, agencias, donantes y demás interesadas en la reconstrucción del servicio educativo y en estrategias de respuesta para aliviar las condiciones de riesgo de la sociedad civil, necesitan mecanismos de coordinación que faciliten la entrega de ayuda de la manera más apropiada, puesto que si esto no se tiene en cuenta, las experiencias demuestran que los programas de intervención no llegan a los directamente afectados, o se encubren unos con otros, haciendo de la ayuda algo poco efectivo en la comunidad afectada.

Por otra parte, las escuelas deben ser respetadas y protegidas como santuarios y zonas de paz; así como los programas de educación han de concebirse de modo que promuevan el pleno desarrollo de la personalidad humana, fortalezcan el respeto de los derechos humanos y las libertades fundamentales proclamados en la Declaración Universal de Derechos Humanos (Artículo 26). Esos programas deben favorecer el entendimiento, la tolerancia y la amistad entre todas las naciones y todos los grupos étnicos y religiosos; han de ser sensibles a las identidades culturales y lingüísticas y respetuosos de la diversidad; $y$, por último, deben reforzar una cultura de paz. Por consiguiente, la educación tiene que promover no sólo la adquisición de habilidades como la prevención y la solución pacífica de los conflictos, sino también valores sociales y éticos.
Esencialmente en Colombia, es recomendable y se hace necesario desarrollar políticas educativas duraderas en las cuales se establezcan planes y programas que actualicen el concepto de la educación como un pilar fundamental para desarrollar procesos que generen equidad, construyan ciudadanías conscientes y activas y disminuyan las brechas de pobreza, la educación debe ser tratada como un derecho fundamental que favorezca a los habitantes más vulnerados socioeconómicamente y debe ser garantizada por el Estado.

El estado colombiano ha tenido avances significativos durante los últimos años, pero es innegable que la tarea está a medio hacer; falta establecer planes y programas que prevean la $\mathrm{cu}$ alificación de los docentes, mejorar la infraestructura, dotación de laboratorios encaminados al desarrollo del emprendimiento, programas de bilingüismo e inclusión de la tecnología en los planes de estudio, escolaridad desde los cuatro años, creación de programas de alimentación, transporte y salud escolar como práctica para disminuir la deserción. La educación debe ser universal y llegar a todos los sectores inclusive los más apartados y vulnerables.

Establecer programas educativos flexibles como una posibilidad para dar oportunidad a las personas que en su momento y como consecuencia del Conflicto no pudieron acceder a programas educativos, es por eso que sin tener en cuenta rangos de edad todas las personas víctimas del conflicto o reinsertados según el caso, tengan acceso a desarrollar currículos flexibles que desarrollen una vocacionalidad técnica o acceso a la educación superior; estos son los hechos y las acciones que conducirán al desarrollo de una paz sostenible, la práctica de los derechos humanos; programas de alfabetización, favorecimiento a la participación de las mujeres.

En general, la enseñanza no debe ser discriminatoria ni segregadora, debe contribuir al progreso de todas las personas garantizando cali- 
dad en todos los aspectos que la contemplan. De esta manera, los sistemas educativos en sociedades posconflicto deben contemplar en sus currículos la neutralidad, la sensibilización y formación en temas de género, promover la participación activa de las mujeres, libertad religiosa, la interdisciplinariedad como factor de promoción humana mediante el aprendizaje de artes $\mathrm{u}$ oficios y quehaceres para la vida.

\section{Referencias}

CEPAL. (2001). La migración internacional y el desarrollo de las Américas. Santiago de Chile: Naciones Unidas.

Chaparro, A. (2009). Génesis y Transformaciones del Estado Nación en Colombia. Bogotá: Universidad del Rosario.

CINEP. (2005). Tercer año de gobierno de Álvaro Uribe Vélez. Más allá del embrujo, 94.

Comité Internacional de la Cruz Roja - CICR. (2005). Derecho Internacional Humanitario. Ginebra Suiza: CICR.

Congreso de la República de Colombia. (1994). Ley 115 de febrero 8 de 1994. Bogotá: ECOE ediciones.

Corte Constitucional. (2016). Constitución Política de Colombia 1991. Bogotá: BELM.

Fundación Dos Mundos. (2009). Escuela y conflicto armado: de bien protegido a espacio protector. Bogotá: Espacio Creativo Impresores.

González, P. (2005). Investigación Educativa y Formación del Docente Investigador. Santiago de Cali: Gráficas Ledesma.

Hernández, R. \& Fernández, C. (2014). Metodología de la investigación. México: Mc Graw Hill Education.

Human Right Watch. (2002). Informe anual Human Right Watch, 2002. New York: Human Right Watch.

INNE. (2004). Normas Mínimas para la Educación en Emergencia: Preparación, Respuesta, Recuperación. New York: INNE.
Malley, A., \& Triplehorn, C. (2005). Educación Emergente en Iraq. Migraciones Forzadas.

Ministerio de Educación Nacional. (2004). Guía $\mathrm{N}^{\circ}$ 8: Guía para la administración de los recursos financieros del sector educativo. Bogotá: MEN.

Ministerio de Educación Nacional. (2008). Grupo Gestor de Educación en Situaciones de Educación en Emergencia. Bogotá: MEN.

Ministerio de Educación Nacional. (2008). Plan Sectorial 2006 - 2010: Revolución Educativa Documento $\mathrm{N}^{\circ} 8$. Bogotá: MEN.

Ministerio de Educación Nacional. (2011). Decisiones acertadas de Educación en Emergencia (módulo 1). Bogotá: RET - the Refugee Education Trust.

Ministerio de Educación Nacional. (2012). Convenio de Cooperación Organización Internacional para las Migraciones (OIM) - MEN. Bogotá: Ministerio de Educación Nacional.

Ministerio de Educación Nacional. (2012). Informe de Eduacación en Emergencia. Bogotá: MEN.

Muñoz, V. (2008). El derecho a la educación en situaciones de emergencia. RAZONES Y EMOCIONES educación en situaciones de emergencia, 19.

Naciones Unidas. (2015). Declaración Universal de los Derechos Humanos. New York: ONU.

Nicolai, S., \& Hine, S. y. (2015). Educación en Emergencias y Crisis Prolongadas. Oslo: Instituto de Desarrollo de Ultramar.

ONU. (2005). Informe sobre Desarrollo Humano 2005. Madrid: Ediciones Mundi-Prensa 2005.

Plataforma Colombiana de Derechos Humanos Democracia y Desarrollo. (2010). Informe Alterno al Quinto Informe del Estado Colombiano Ante el Comité de Derechos Económicos, Sociales y Culturales. Bogotá: Gente Nueva Editorial.

Procuraduría General de la Nación. (2010). Código de la Infancia y la Adolescencia. Bogotá: Graficov Ltda. 
Restrepo Yusti, M. (2008). Apuntes para una reflexión sobre pedagogía de los derechos humanos en Colombia. Chile: OEI.

Sibbons, M. (2004). DFID Case Study 5: The Delivery Of Education Services In Difficult Circumstances: The case Of Nigeria and Sirilanka. DFID: Healt Systems Resource Centre (pág. 10 y 13). London: DFID.

Tiempo, E. (20 de mayo de 2018). ElTiempo. com . Obtenido de http://www.eltiempo. com/multimedia/especiales/refugiadosmigracion-y-desplazamiento-de-colombianos/15503358/1/index.html
Triplehorn, C. (2001). Education. Care \& Protection of Children in Emergencies a Field Guide. Connecticut: Save the Children Federation.

Uprimny Yepes, R. (2004). Orden Democrático y Manejo de Conflictos. Bogotá: Servigraphic Ltda.

Van Dijk, T. (1999). El Análisis Crítico del Dicurso. Anthropos, 26 - 36.

Weiss Fagen, P., Fernández Juan, A., \& Stepputate, F. y. (2009). Internal displacement in Colombia. Refuge, 2(26), 114. 